\title{
Utilização de Flexquest como TDIC para o Ensino de Reações Orgânicas no Ensino Médio
}

\author{
Bárbara L. de Oliveira da Silva ${ }^{1}$, Ariane N. dos Santos ${ }^{1}$, Kátia A. da Silva Aquino ${ }^{2}$ \\ ${ }^{1}$ Departamento de Química Fundamental / Grupo de Estudos e Pesquisa sobre \\ Aprendizagem Significativa (GEPAS) - Universidade Federal de Pernambuco (UFPE) \\ ${ }^{2}$ Colégio de Aplicação/ Programa de Pós-Graduação em Rede Nacional para o \\ Ensino de Ciências Ambientais/ Grupo de Estudos e Pesquisa sobre Aprendizagem \\ Significativa (GEPAS) - Universidade Federal de Pernambuco (UFPE) \\ barbarallucialgmail.com, arianesantosnas@gmail.com, aquino@ufpe.com
}

\begin{abstract}
The advance through scientific and technological progress of the society claims from the schools to acquire new advanced tools. This article intends to analyze an application of a technological device, Flexquest, about diets theme for teaching organic chemical reactions in the last year of high school, from the perspective of the theory of cognitive flexibility. The results present the promotion of applied and useful knowledge in solving students' daily problems, attaining complex knowledge.
\end{abstract}

Resumo. O progresso científico e tecnológico da Sociedade exige do contexto educacional a utilização de ferramentas alinhadas a estes avanços. O trabalho realizado trata da aplicação de uma ferramenta tecnológica, a Flexquest, sobre a temática de dietas para o ensino de reações orgânicas no $3^{\circ}$ ano do ensino médio a partir da perspectiva da teoria da flexibilidade cognitiva. Os resultados apontam para a promoção de um conhecimento aplicável em diversas situações e útil na resolução de problemas do cotidiano dos estudantes, possibilitando o domínio de um conteúdo complexo.

\section{Introdução}

O conjunto de aprendizagens essenciais a serem desenvolvidas na educação básica em química descreve competências que objetivam o desenvolvimento de conhecimentos, habilidades, atitudes e valores que despertem no estudante a capacidade de resolver demandas complexas da vida cotidiana e da sociedade [Brasil 2018]. Para isso, o ensino tradicional pautado em uma aprendizagem mecanizada dever ser superado. Tratando-se da química orgânica, Mariano (2008) explica que esse conteúdo ainda é abordado de forma muito expositiva e fragmentada, completamente distante de uma perspectiva contextualizada e interdisciplinar.

A utilização de Tecnologias Digitais da Informação e Comunicação (TDICs) pode ser uma estratégia bastante eficiente para processos de ensino-aprendizagem mais construtivistas [Fontana 2015]. As TDICs são conceituadas como sendo diversas ferramentas tecnológicas e digitais, como internet, computadores, celulares, plataformas educacionais ou qualquer tipo de hipermídia. Oliveira (2015) atenta para o fato de que TDICs podem promover uma compreensão ampla e crítica de um determinado conteúdo, conforme este é representado através de diferentes perspectivas, de forma a flexibilizá-lo [Moran 2000].

Proposta por Spiro e colaboradores (1988), a Teoria da Flexibilidade Cognitiva (TFC) alega que para o entendimento profundo de conhecimentos complexos, estes, 
devem ser trabalhados através de diversas explicações, visões, analogias, isto é, múltiplas representações. Isso significa dizer, que a TFC defende que o conhecimento deve ser utilizado de diversas formas, buscando a resolução de problemas do dia-a-dia, através de casos que interagem entre si, promovendo a contextualização e a interdisciplinaridade [Carvalho 2011]. Para Aleixo (2008) as TDICs podem ser uma estratégia de aplicação da TFC. Articulado a isso, a Flexquest (FQ), um exemplo de TDICs, se caracteriza como um instrumento metodológico, que utiliza a internet para obter informações diversas, através de variados contextos, proporcionando um processo de ensino mais crítico e mais flexível [Aleixo 2008].

O presente trabalho apresenta em sua fundamentação teórica uma breve discussão acerca do ensino de química com foco nas reações orgânicas, além disso, faz um paralelo com as Tecnologias Digitas da Informação e Comunicação (TDICs). Por fim, demonstra como a ferramenta Flexquest se articula à Teoria da Flexibilidade Cognitiva. A metodologia consiste na descrição de uma sequência didática com a temática de dietas da moda e relata a aplicação da plataforma Flexquest como recurso didático cujo conteúdo subsidiou a socialização das atividades desenvolvidas em grupo que gerou a produção de um caso, elemento solicitado no tópico de transferência da plataforma. A análise dos resultados, de forma qualitativa, foi realizada através da avaliação das atividades propostas a partir da Flexquest: as questões, os processos e a transferência. Nesse sentido, o objetivo desse trabalho é apresentar uma sequência didática, abordando o conteúdo de Reações Orgânicas, de forma contextual e interdisciplinar, utilizando a ferramenta tecnológica Flexquest como facilitadora de uma aprendizagem inserida na perspectiva da Teoria da Flexibilidade Cognitiva.

\section{Fundamentação Teórica}

\subsection{Ensino de Química no Século XXI e as Reações Orgânicas}

Os Parâmetros Curriculares Nacionais do Ensino Médio destacam que a Química "[...] deve possibilitar ao aluno a compreensão tanto dos processos químicos em si, quanto da construção de um conhecimento científico em estreita relação com as aplicações tecnológicas e suas implicações ambientais, sociais, políticas e econômicas" [Brasil 2002 p.87]. O ensino de Química, no século XXI, deve ser essencial para compreender os "fenômenos do mundo natural e tomar decisões políticas e sociais que possibilitem a vivência democrática, cidadã e humana com dignidade" [Scheid 2016 p. 281] que promovam ao estudante "elaborar pensamentos autônomos e críticos e formular os seus próprios juízos de valor, de modo a poder decidir, por si mesmo, como agir nas diferentes circunstâncias da vida" [Delors 1998 p. 99].

Esta proposta se opõe às práticas de ensino tradicionais de memorização de informações, e aplicação de conhecimento fragmentados e monodisciplinares. Entretanto, segundo Binsfeld (2013), ao tratar dos assuntos referentes à Química Orgânica, por exemplo, os estudantes relatam que as aulas de desenvolvem no processo de memorização do conteúdo e repetição de exercícios, reverberando na falta de interesse dos alunos e na dificuldade dos mesmos em compreender os impactos da ciência na sociedade. Já Mariano (2008) argumenta que a compreensão dos estudantes em relação às reações orgânicas indica um conhecimento compartimentalizado e vinculado à 'intuição'. 
Espera-se das abordagens pedagógicas de Química que os estudantes compreendam os processos químicos e suas correlações com os diversos sistemas estruturais da sociedade, tais como o agrícola, tecnológico, científico e produtivo; através de práticas pedagógicas diferenciadas e enviesadas com os eixos da contextualização e interdisciplinaridade [Brasil 2002]

\subsection{Tecnologias Digitais da Informação e Comunicação}

Diante dos diversos avanços científicos e tecnológicos que a sociedade tem enfrentado, cabe a escola adequar seus métodos de ensino através da inclusão de tecnologias digitais que auxiliem no aprimoramento do aprendizado dos estudantes. Tendo em vista que os educandos da rede básica têm cada vez mais se apropriado de tecnologias mais avançadas [Rezende 2017] e que estas ferramentas têm caráter lúdico, dinamismo e maior potencial para os processos de ensino-aprendizagem. [Fontana e Cordenonsi 2015]

Segundo Tavares e colaboradores (2013 p. 156), as Tecnologias de Informação e Comunicação (TICs) são denominadas como "um conjunto de recursos tecnológicos que podem proporcionar comunicação e/ou automação de diversos tipos de processos em diversas áreas e principalmente no ensino e na pesquisa". Atualmente, as TICs foram atualizadas para Tecnologias Digitais de Informação e Comunicação (TDICs) designando a inserção de elementos digitais, isto é, os aparelhos eletrônicos mais avançados e os recursos que os mesmos disponibilizam, tais como a internet, softwares e plataformas educacionais.

Dentre as vantagens das TDICs no âmbito educacional, pode-se considerar que, normalmente, se adequam às distintas necessidades dos estudantes através da pluralidade de recursos didáticos que disponibilizam e ainda permitem ao professor apresentar a informação de maneira variada. Ao serem associadas a uma proposta pedagógica estruturada e aplicadas nos processos de aprendizagem, tornam-se dispositivos mediadores do saber [Oliveira 2015] e podem possibilitar a flexibilização no tratamento das informações e, consequentemente, o entendimento amplo do conhecimento através das múltiplas representações que incorpora; a exemplo do texto, imagem, som e video [Moran 2000]. A utilização das TDICs nas práticas pedagógicas facilita a assimilação do conhecimento, estimulam as atividades cooperativas e o desenvolvimento do senso crítico dos educandos.

\subsection{Teoria da Flexibilidade Cognitiva e Flexquest}

Rand Spiro e colaboradores constataram, no final dos anos 80 (através de pesquisas com turmas de medicina), que estudantes tinham dificuldades em transferir e aplicar alguns conhecimentos em situações alternativas ao âmbito acadêmico. Eles verificaram uma tendência desses estudantes de simplificar conteúdos complexos e, consequentemente, na maioria das vezes, alcançar compreensões enganosas. Spiro (1988) argumenta, então, que para uma aprendizagem que visa o domínio de conteúdos complexos, deve-se conseguir utilizá-los em diversos contextos. Dessa forma, são necessárias múltiplas representações, explicações, comparações; de forma a flexibilizar o conhecimento, e assim, compreendê-lo profundamente [Spiro 1988, Pessoa e Nogueira 2009].

A partir dessa perspectiva, Spiro e colaboradores desenvolveram a Teoria da Flexibilidade Cognitiva (TFC), que se conceitua como sendo a capacidade de aplicação de conhecimentos complexos em diversas situações. Carvalho (2011) atenta para o fato 
de que através da TFC os processos de aprendizagem por memorização são superados e o estudante consegue, por exemplo, utilizar o conhecimento para resolução de problemas cotidianos. Por meio da TFC conhecimentos complexos são retratados em múltiplas representações, temas, analogias e dimensões, através de casos que se articulam e estabelecem conexões entre si [Carvalho 1999 apud Vasconcelos 2012].

No sentido de utilizar o conhecimento de diversas formas, as Tecnologias Digitais de Informação e Comunicação (TDICs) se apresentam como um meio bastante eficiente que viabiliza a aplicação da TFC. As TDICs são ferramentas como computadores, internet, aparelhos de celular, hipermídias, em geral [Aleixo 2008]. Spiro e Jehng (1990) ressaltam o fato das hipermídias proporcionarem várias representações de um mesmo conteúdo em diversos níveis, inclusive os mais avançados, dessa forma, as TDICs possibilitam que o conhecimento seja trabalhado através de variadas perspectivas [Pessoa e Nogueira 2009].

A Webquest (WQ) é um exemplo que identifica as TDICs. Definida como uma metodologia de ensino de atividade de pesquisa, mediada pelo professor, que utiliza, predominantemente, informações obtidas da internet. A WQ sofreu algumas alterações e a Flexquest (FQ) ou Webquest de segunda geração, é uma versão mais avançada dessa metodologia, baseada na TFC. Isso significa que a FQ se caracteriza como uma ferramenta capaz de promover a aplicação de conteúdos complexos em diferentes contextos, ou seja, proporcionando sua flexibilização [Aleixo 2008].

Segundo Santos (2016), a FQ se identifica na estruturação de contextos baseados na realidade. Isto é, casos que se dividem em unidades menores e, assim, possibilitam a compreensão avançada do conteúdo complexo. A utilização da FQ promove um ensino não linear, relacional e multidimensional que objetiva a construção flexível do conhecimento.

\section{Metodologia}

O presente estudo foi realizado no Colégio de Aplicação da Universidade Federal de Pernambuco (CAp-UFPE), na cidade do Recife, durante as aulas de Química de duas turmas do $3^{\circ}$ ano do ensino médio, somando um total de 60 alunos. A pesquisa teve cunho qualitativo, isto é, as análises foram feitas através das observações dos estudantes durante o desenvolvimento das aulas e das atividades realizadas a partir da Flexquest. A metodologia está descrita numa sequência didática de quatro etapas sintetizadas na Tabela 1.

Os momentos da sequência foram conduzidos com a participação da docente do componente curricular de Química da turma com auxílio de duas licenciandas (autoras desse trabalho). No primeiro momento (aulas de caráter teórico), o conteúdo de reações orgânicas foi apresentado à turma, primeiramente, definindo os tipos de reações orgânicas e explicando as equações químicas características de cada uma, utilizando exemplos cotidianos. Depois, as reações orgânicas foram classificadas com seus respectivos mecanismos e os tipos de cisão foram demonstrados. Algumas atividades para identificação dos tipos de reações foram realizadas e questionários auto avaliativos foram aplicados no fim de cada aula para que os estudantes realizassem uma autoavaliação. 
Tabela 1. Sequência didática trabalhada neste estudo.

\begin{tabular}{|c|c|c|c|}
\hline & Aulas & Atividades & Recursos \\
\hline $\begin{array}{c}\text { Primeiro } \\
\text { Momento }\end{array}$ & 6 horas/aula & $\begin{array}{l}\text { Caracterização dos tipos de reações orgânicas } \\
\text { (adição, eliminação, oxidação, substituição e } \\
\text { complexação) e seus mecanismos; Definição } \\
\text { dos tipos de cisão através de discussão sobre } \\
\text { protetores solares e os radicais livres. }\end{array}$ & $\begin{array}{l}\text { Slides; } \\
\text { computador; } \\
\text { projetor. }\end{array}$ \\
\hline $\begin{array}{l}\text { Segundo } \\
\text { Momento }\end{array}$ & 2 horas/aula & $\begin{array}{l}\text { Introdução à plataforma Flexquest (produzida } \\
\text { pelo grupo Semente da Universidade Federal } \\
\text { Rural de Pernambuco - UFRPE), discussão } \\
\text { da seção dos "casos" presente na Flexquest } \\
\text { "Tem química na minha dieta?"1 }\end{array}$ & $\begin{array}{c}\text { Slides; } \\
\text { computador; } \\
\text { projetor; } \\
\text { smartphones; } \\
\text { plataforma } \\
\text { Flexquest. }\end{array}$ \\
\hline $\begin{array}{c}\text { Terceiro } \\
\text { Momento }\end{array}$ & 8 horas/aula & $\begin{array}{c}\text { Abordagem conceitual sobre bioquímica dos } \\
\text { macronutrientes: carboidratos, lipídeos e } \\
\text { proteínas, no organismo e sua relação com as } \\
\text { dietas; Análise do ciclo de Krebs e da beta } \\
\text { oxidação dos lipídeos; Aplicação de exercícios } \\
\text { envolvendo classificação de reações } \\
\text { orgânicas e identificação das rotas } \\
\text { metabólicas. }\end{array}$ & $\begin{array}{c}\text { Slides; } \\
\text { computador; } \\
\text { projetor. }\end{array}$ \\
\hline $\begin{array}{c}\text { Quarto } \\
\text { Momento }\end{array}$ & 4 horas/aula & $\begin{array}{c}\text { Socialização da seção de transferência da } \\
\text { Flexquest e entrega das atividades escritas } \\
\text { (questões, processos e transferência); } \\
\text { apresentação dos grupos da seção } \\
\text { transferência, fazendo articulação, numa } \\
\text { perspectiva interdisciplinar, do conteúdo de } \\
\text { reações orgânicas com o componente } \\
\text { curricular de Educação Física (para tratar de } \\
\text { atividades físicas na temática de dietas) com } \\
\text { posterior discussão. }\end{array}$ & -- \\
\hline
\end{tabular}

$\mathrm{Na}$ apresentação da plataforma Flexquest (segundo momento), os estudantes, divididos em quatro grupos, usaram seus aparelhos de smartphone para acessar a página da plataforma Flexquest que tem como tema as Dietas (low carb, paleolítica, Dukan e cetogênica). Nessa aula, os estudantes de cada grupo (um grupo para cada dieta) tiveram que ler os textos e assistir os vídeos contidos na Flexquest, correspondente a sua dieta, e apresentar para a turma suas considerações, conceituando e detalhando o tipo de dieta.

No terceiro momento, também caracterizado por aulas mais teóricas, ocorreu a articulação entre as reações orgânicas e as dietas, a partir de uma abordagem bioquímica, tratando dos macronutrientes (carboidratos, lipídeos e proteínas) e de processos orgânicos como o ciclo de Krebs e o de Lynen (ou da beta oxidação dos lipídeos), nos quais as reações orgânicas estão presentes.

O quarto momento, constituiu-se pela entrega das questões e dos processos, além da socialização da parte da transferência da Flexquest. O trabalho exigia que cada grupo escolhesse uma notícia que relatasse um tipo de dieta (que o grupo ficou

${ }^{1}$ Tem química na minha dieta? -disponível em<http://flexquest.ufrpe.br/projeto/2687/caso/> 
responsável) e uma atividade física. $\mathrm{Na}$ apresentação, os estudantes deveriam articular conteúdos de Química, Bioquímica e Educação Física discutindo a eficiência da dieta para a respectiva atividade física. Neste último momento, houve também a participação da Professora de Educação Física da turma que contribuiu com a socialiação dos estudantes.

\section{Resultados e Discussão}

A aplicação da sequência didática proporcionou aos estudantes maior interesse em compreender os conceitos científicos e engajamento, em especial, ao utilizar a ferramenta Flexquest, corroborando com a afirmação de que a plataforma tecnológica facilita os processos de aprendizagem. De acordo com Rezende (2017) é trivial a utilização de instrumentos diferentes e de cunho tecnológico no âmbito escolar, alinhados ao progresso da sociedade. Aleixo (2008) indica que esse instrumento unido a uma estratégia planejada e bem estruturada se tornam ferramentas com bastante potencial de ensino. O mesmo enfatiza Fontana e Cordenonsi (2015) ao reconhecer que promovem também ludicidade e dinamismo nas atividades.

De modo geral, a Flexquest Dietas é subdivida pelo contexto das dietas restritivas; os casos que são os tipos de dietas da moda (dukan, paleolítica, cetogênica e low carb); as questões que são perguntas objetivas sobre os casos; processos, perguntas que envolvem casos entre si; e a transferência, momento de socialização de uma atividade promovida pelo docente. A figura 1 seguinte ilustra a página da Flexquest na parte referente aos casos que envolvem cada dieta:

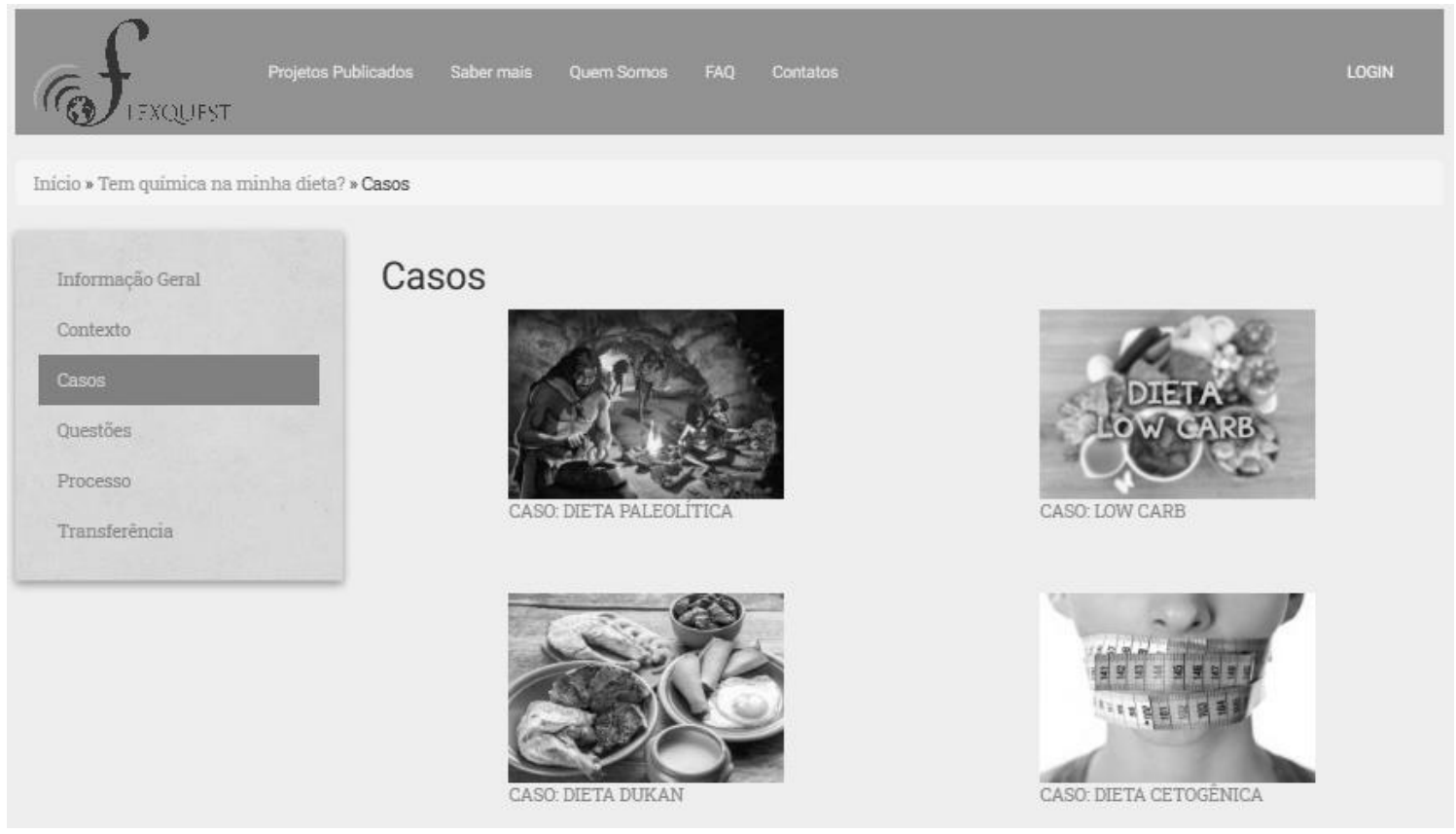

Figura 1. Página da plataforma Flexquest apresentando os casos sobre as dietas.

Os casos contêm informações oriundas da internet que caracterizam e definem as dietas. Estas informações são apresentadas em forma de texto ou imagem/vídeo, isto é, a partir de múltiplas representações de conhecimentos complexos [Vasconcelos 2012]. Pode-se reconhecer que os casos associados à Flexquest enquanto TDIC 
permitiram a flexibilização e compreensão de conhecimento avançado, multirepresentacional e aplicável às diferentes vivências de mundo do estudante. [Moran 2000 e Santos 2016]

A análise da proposta didática foi realizada a partir das respostas das questões, processos e transferência de um dos grupos de estudantes participantes. As questões remetem-se a quatro perguntas que envolvem implicitamente os casos e minicasos às reações orgânicas. O grupo caracterizou cada dieta; e esclareceu a diferença entre as dietas low carb e cetogênica em relação à quantidade de carboidratos, a importância dos corpos cetônicos no organismo e os males causados pelo excesso de corpo cetônicos nestas dietas; e das proteínas na Dukan que podem resultar em cálculo renal. Faltando apenas comentar as fases da dieta Dukan segundo a proporção dos macronutrientes e relacionar às reações orgânicas ao ciclo de Krebs. Não obstante, explicaram rotas metabólicas e conteúdos que não viram durante a aula, indicando que conseguiram se aprofundar e pesquisar além do proposto em sala.

O grupo também salientou que a dieta paleolítica é a mais apropriada para uma alimentação equilibrada e, assim, reconheceu a importância da proporção balanceada de macronutrientes no organismo segundo as orientações médicas para evitar riscos à saúde. Isto indica que os estudantes puderam desenvolver "pensamentos autônomos e críticos" [Delors 1998 p. 99] e tomar suas próprias conclusões quanto às consequências das dietas no corpo. A produção textual sobre as questões continha articulações claras e bem estruturadas, incluindo um tópico sobre a elaboração de uma matéria jornalística para discutir as questões socioeconômicas relacionadas às dietas restritivas. $\mathrm{O}$ grupo compreendeu a estratégia lucrativa da indústria da moda das dietas e dissertou objetivamente sobre. De modo geral, o grupo conseguiu contextualizar as reações orgânicas às rotas metabólicas e às dietas, tal como alcançar a dimensão interdisciplinar por discutir conteúdos tanto de Química como de Biologia, obtendo resultados bastante satisfatórios do objetivo das questões e ainda alcançando eixos pedagógicos pertinentes à educação química esperada para o século XXI [Brasil 2002].

Os processos também apresentam quatro problemas que envolvem implicitamente as reações orgânicas, entretanto articulam os diferentes minicasos das dietas entre si para desafiar os educandos a partir de outro ponto de vista, como afirmam Pessoa e Nogueira (2009) que as TDICs apresentam a propriedade de trabalhar o conhecimento a partir de diversas perspectivas. Os estudantes puderam elencar as razões sociais que implicam a alta demanda das dietas na atualidade, como a pressão social pelo "corpo perfeito" e altas expectativas criadas que são estratégias da indústria das dietas para atrair adeptos. Porém o grupo não detalhou suficientemente rotas alternativas tal como a produção de corpos cetônicos e a gliconeogênese que podem ocasionar o sobrecarregamento de alguns órgãos e consequentes males à saúde.

Mesmo assim, pode-se inferir que o processo de flexibilização cognitiva estava ocorrendo e ampliando a partir das ricas articulações e posicionamentos críticos dos alunos sobre os problemas relacionados às dietas em seu cotidiano [Carvalho 2011]. De acordo com os Parâmetros Curriculares Nacionais do Ensino Médio [Brasil 2002], a Química deve promover o entendimento dos processos químicos relacionados às aplicações tecnológicas e questões sócio-políticas e econômicas, isto é, o conhecimento científico deve ser vinculado à realidade do estudante. 
O intuito dos processos foi de promover a interação entre os minicasos dos diferentes casos para o desenvolvimento do aprendizado em domínios complexos. Desta forma, mesmo faltando algumas informações importantes, os estudantes demonstraram processos cognitivos avançados, mesmo que em algumas ocasiões não tenham sido ampliados, foram flexibilizados. [Spiro 1988]

A transferência visa a proposta de uma atividade pelo professor para que os estudantes, em grupo, consigam aplicar o conteúdo em outro contexto e flexibilizar o conhecimento. Enquanto ferramenta tecnológica, a Flexquest pôde ainda estimular o trabalho cooperativo, um dos benefícios das TDICs. Além de permitir a mediação entre os saberes do estudante, do docente e o conhecimento em si [Aleixo 2008 e Oliveira 2005]. Foi proposto aos estudantes a escolha por uma matéria jornalística que abordasse dois minicasos no mínimo (atividade física e dieta) para articular criticamente, a partir da perspectiva interdisciplinar, conceitos de educação física e bioquímica que justificassem a adequação da dieta à atividade física citada na matéria.

O grupo percebeu que a matéria apresenta um título chamativo; cita a entrevista de uma nutricionista para transparecer confiança na informação sobre a dieta e a atividade física, porém não aborda os possíveis malefícios da dieta e nem orienta quanto à necessidade de um acompanhamento médico. Assim, concluem que a matéria é atrativa e sensacionalista para enaltecer a indústria de dietas e lucrar, como haviam discutido na seção das questões.

Mesmo com as deficiências de conteúdo da matéria jornalística, os estudantes articularam os movimentos "primevos" da atividade física de crossfit à alimentação "primitiva" da dieta paleolítica, atividade e dieta abordados na notícia, respectivamente. Também vincularam, de maneira interdisciplinar, os conceitos de educação física, como a individualidade biológica, um dos princípios do treinamento físico; às reações orgânicas, à bioquímica e aos riscos de adesão das dietas da moda. Esse intercâmbio entre áreas de conhecimento indicam o nível de aprofundamento e flexibilização que os estudantes conseguiram realizar em sua estrutura cognitiva [Spiro 1988, Pessoa e Nogueira 2009] para a compreensão avançada de conteúdos complexos ofertados pela plataforma tecnológica Flexquest [Santos 2016].

A apresentação da Flexquest foi encerrada com a confraternização entre os docentes de Química e Educação Física, e os estudantes das turmas que ficaram responsáveis de trazer alimentos que mais se adequavam a dieta articulada pelo grupo na seção de transferência, a exemplo de bacon, omelete, frutas, chá e amendoim.

\section{Considerações Finais}

De acordo com os Parâmetros Curriculares Nacionais do Ensino Médio (2002) o ensino de química deve propiciar o entendimento das transformações e fenômenos químicos naturais e tecnológicos, com o objetivo de agir, de forma crítica, em diversos contextos sociais. Essa perspectiva não é contemplada por práticas de ensino tradicionais, como a transferência e a memorização. O conteúdo de reações orgânicas, foco desse estudo, tão importante por discutir processos intrínsecos à vida, é, frequentemente, estudado de modo fragmentado e incompleto, assim como aponta Mariano (2008), por ser considerado um assunto denso e complexo. Por esse motivo, documentos oficiais como os PCNEMs, atentam para a necessidade de se diversificar práticas docentes, que devem 
ser sempre pautadas da contextualização e interdisciplinaridade, tratando do viés social e tecnológico.

A utilização das TDICs é compreendida por Rezende (2017) como uma ferramenta que amplia o potencial de aprendizagem, visto que, instrumentos tecnológicos estão cada vez mais inseridos no contexto escolar. Além de ser um instrumento que diversifica práticas pedagógicas, as TDICs podem auxiliar na flexibilização de conhecimentos complexos, por representá-los através de textos, imagens, sons e vídeos [Moran 2000].

Para que conteúdos complexos (como muitas vezes é considerado o tópico de reações orgânicas) sejam compreendidos de forma ampla e profunda, deve-se conseguir aplicá-los em várias representações. Em função disso, a aplicação da ferramenta Flexquest, no âmbito educacional, confirmou sua eficiência enquanto TDIC por facilitar o aprendizado de reações orgânicas e promover posicionamento crítico sobre os desafios enfrentados pelos estudantes sobre o mundo das dietas; além de flexibilizar e ampliar o conhecimento ao aplicá-lo na resolução de problemas presentes na realidade dos educandos.

\section{Referências}

Aleixo, A. A., Leão, M. B. C. e Souza, F. N. (2008) "FlexQuest: potencializando a WebQuest no Ensino de Química", R. Faced, n.14, p. 119 a 133.

Binsfeld, S. C., Auth, M. A. e Macêdo, A. P. A (2013) "Química Orgânica no Ensino Médio: evidências e orientações", Universidade Federal do Rio De Janeiro. Núcleo de Tecnologia Educacional para a Saúde. Atas do IX Encontro Nacional de Pesquisa em Educação em Ciências - IX ENPEC. Águas de Lindóia.

Brasil. Ministério da Educação (MEC) - Secretaria de Educação Média e tecnologia (Semtec) (2002). "PCN+ Ensino Médio: Orientações Educacionais Complementares aos Parâmetros Curriculares Nacionais - Ciências da Natureza, Matemática e suas Tecnologias", Brasília: MEC/SEMTEC.

Brasil. Ministério da Educação. (2018) "Base nacional comum curricular", http://basenacionalcomum.mec.gov.br/images/BNCC_EI_EF_110518_versaofinal_si te.pdf, Junho.

Carvalho, A. A. A. (2011) “Teoria da Flexibilidade Cognitiva e o Modelo Múltiplas Perspectivas",https://repositorium.sdum.uminho.pt/bitstream/1822/15921/1/Carvalho \%202011\%20-\%20TFC\%20e\%20MMP.pdf, Outubro.

Delors, J. (1998) "Educação: um tesouro a descobrir", $3^{\text {a }}$ edição. São Paulo: Cortez, Brasília-DF: MEC-UNESCO.

Fontana, F. F. e Cordenonsi, A. Z. (2015) "TDIC como mediadora do processo de ensino-aprendizagem da arquivologia", Ágora, Florianópolis, v. 25, n. 51, páginas 101-131, jul./dez.

Mariano, A. (2008) "O ensino de reações orgânicas usando química computacional: I. Reações de adição eletrofílica a alquenos”, Química Nova. v. 31, n. 5, páginas 1241249. 
Moran, J. M., Masetto, M. T., Behrens, M. A. (2000) "Novas tecnologias e mediação pedagógica", $13^{\mathrm{a}}$ edição. Campinas: Papirus.

Oliveira, C. (2015) "TIC's na educação: A utilização das tecnologias da informação e comunicação na aprendizagem do aluno", Pedagogia em Ação. v. 7. n. 1, páginas 7595.

Pessoa, T., Nogueira, F. (2009) "Flexibilidade cognitiva nas vivências e práticas educativas", Educação e contemporaneidade: pesquisas científicas e tecnológicas. EDUFBA, Salvador, 400 p.

Resende, D. V. (2017) "Uso Criativo das Tecnologias da Informação e Comunicação na Educação Superior: atuação de professores e percepção de estudantes”, $237 \mathrm{f}$. Tese (Doutorado em Processos de Desenvolvimento Humano e Saúde). Universidade de Brasília, Brasília.

Santos, I. G. S. (2016) "FLEXQUEST: Uma plataforma Web 2.0 para o desenvolvimento de atividades interdisciplinares visando à promoção de flexibilidade cognitiva", UFRPE. Tese de Doutorado.

Scheid, N. M. J. (2016) "Os desafios da docência em ciências naturais no século XXI", Rev. Fac. Cienc. Tecnol. [online]. n.40, páginas 277-309.

Spiro, R. J. e Jehng, J. C. (1990) "Cognitive flexibility and hypertext: theory and technology for the nonlinear and multidimensional traversal of complex subject matter", In; Nix, D., Spiro, R. J. (Ed.). Cognition, education, and multimedia: Cognition, education, and multimedia exploring ideas in high technology. Hillsdale: Lawrence Erlbaum Associates. páginas 163-205.

Spiro, R., Coulson, R., Feltovich, P. e Anderson, D. (1998) "Cognitive flexibility: Advanced knowledge acquisition ill-structured domains", Tenth Annual Conference of the Cognitive Science Society Proceedings. Hillsdale, NJ: Erlbaum, chapter 22, páginas 544 a 557.

Tavares, R., Souza, R. O. O. e Correia, A. O. (2013) "Um estudo sobre a TIC e o Ensino de Química”, Revista GEINTEC, v. 3, n. 5, páginas 155-167, São Cristóvão/SE.

Vasconcelos, F. C. G. C. (2012) "Utilização de recursos audiovisuais em uma estratégia flexquest sobre radioatividade", Investigações em Ensino de Ciências. Revista. v.17, n.1, páginas 37 a 58 . 